\title{
Evaluation of the Effect of Nickel Clusters on the Formation of Incipient Soot Particles from Polycyclic Aromatic Hydrocarbons through ReaxFF Molecular Dynamics Simulations
}

\author{
Sharmin Shabnam ${ }^{1}$, Qian Mao², Adri C.T. van Duin ${ }^{1 *}$, K. H. Luo ${ }^{2,3}$ \\ ${ }^{1}$ Department of Mechanical and Nuclear Engineering, The Pennsylvania State University, \\ University Park, PA 16802, USA \\ ${ }^{2}$ Center for Combustion Energy, Key Laboratory for Thermal Science and Power Engineering of \\ Ministry of Education, Department of Thermal Engineering, Tsinghua University, Beijing \\ 100084, China \\ ${ }^{3}$ Department of Mechanical Engineering, University College London, Torrington Place, London \\ WC1E 7JE, UK \\ *Corresponding author: Email: acv13@psu.edu (Adri van Duin)
}

\begin{abstract}
In the present study, the ReaxFF reactive molecular dynamics simulation method was applied to investigate the effect of a small nickel cluster $\left(\mathrm{Ni}_{13}\right)$ on the formation of nascent soot from polycyclic aromatic hydrocarbon (PAH) precursors. A series of NVT simulations were performed for systems of a $\mathrm{Ni}_{13}$ cluster and various PAH monomers, namely, naphthalene, anthracene, pyrene, coronene, ovalene, and circumcoronene, at temperatures from 400 to $2500 \mathrm{~K}$. At low temperatures, the PAHs form soot particles through binding and stacking around nickel clusters. Larger soot particles are formed due to the early initiation of clustering provided by nickel than those observed in homogenous PAH systems. At 1200 1600 K, the PAH monomers show chemisorption tendency onto the nickel surface, which results in incipient soot particles. Chemical nucleation was observed at $2000 \mathrm{~K}$ where nickel-assisted dehydrogenation and chemisorption of PAH lead to the growth of stable soot particles, which did not occur in the absence of Ni-clusters. At a high temperature $(2500 \mathrm{~K})$, nickel significantly accelerates the ring-opening and graphitization of PAH molecules and increases the size of the fullerene-type soot as compared to that of homogenous systems.
\end{abstract}

\section{Keywords:}

Polycyclic aromatic hydrocarbon (PAH), Soot, Nickel cluster, ReaxFF, Molecular dynamics (MD) 


\section{Introduction}

The fundamental mechanism of soot formation draws substantial interest from the research communities on combustion, energy and the environment. In addition to its adverse effect on engine fuel efficiency ${ }^{1}$, soot is regarded as one of the major environment contaminants ${ }^{2}$. It has been positively identified as the leading cause of respiratory and cardiovascular disease $\mathrm{e}^{3,4}$ particularly in urban areas $^{5}$ posing a great risk to human lives. However, soot produced in controlled environment (carbon black) is used commercially in the production of automotive tires and as a component of paints and toners in copy machines and laser printers ${ }^{6}$, or more recently, in photovoltaic solar cells ${ }^{7}$.

The detrimental effects of soot, as well as its useful applications demand a thorough knowledge of the underlying mechanism of soot inception and growth. The conversion of gasphase hydrocarbon fuel molecules to solid carbonaceous agglomerates involves many fast and concurrent reactions within a few milliseconds. Numerous experiments and modeling studies reveal four major processes of soot formation such as formation of aromatics, nucleation or inception of nascent soot particles, particle growth by coagulation, surface reactions (growth and oxidation $)^{8-10}$. The current description of sooting mechanism is, however, incomplete due to the difficulty in capturing the complex nature and the large number of inter-related elementary pathways involved in the combustion of real fuels. At present, the majority of the experimental and modeling studies recognize the central role of gaseous polycyclic aromatic hydrocarbon (PAH) precursors in the formation of solid soot particles ${ }^{9,11}$. The early models assume the growth of soot resulted only from chemical reactions ${ }^{8}$. Later studies by Frenklach et al. showed the formation of condensed soot particle through physical stacking of PAHs ${ }^{12}$. 
A significant aspect of sooting mechanism which is still not well understood is the effect of metal particles on the mechanism of soot nucleation. Lough et al. (2005) studied metal emissions from motor vehicles with inductively-coupled plasma mass spectrometry and found that $19 \%$ of particulate matters $\left(\mathrm{PM}_{10}\right)$ were consisted of metals such as $\mathrm{Si}, \mathrm{Fe}, \mathrm{Ca}, \mathrm{Na}, \mathrm{Mg}, \mathrm{Al}$, and $\mathrm{K}^{13}$. Lombart et al. (2004) used Laser Induced Breakdown Spectroscopy to analyze diesel engine exhaust particles and identified $\mathrm{Fe}, \mathrm{Cu}, \mathrm{Ca}, \mathrm{Zn}$ and $\mathrm{Mg}$ and sulfur as major metallic species ${ }^{14}$. Corbin et al. (2018) identified toxic heavy metals such as V and Ni in the PM emission of a marine diesel engine using soot-particle aerosol mass spectrometry (SP-AMS) ${ }^{15}$. Presence of these inorganic elements in vehicle exhausts is mostly attributed to metals or metallic additives in fuel ${ }^{16}$ and lubricating oil additives ${ }^{17}$ or engine wear ${ }^{18}$. Fuel additives have been widely used in diesel or biofuel combustion to combat pollutant emission or to increase ignition or combustion efficiency. The metals were observed to manifest soot enhancement or suppression capability by altering the soot size, number density and volume fraction. Ferrocene, an iron based fuel additive has been shown to enhance the amount of soot formed including both soot particle size and volume fraction in primary flame zones ${ }^{19,20}$. The iron compounds dissociate well before soot inception and act as iron-rich nuclei that provide sufficient surface area for soot formation and growth. Later, in the soot oxidation or burnout stage, iron oxides show soot suppression mechanism through catalytic oxidation thereby reducing soot emission. Similar results of both soot-enhancing and suppressing characteristics were found with iron pentacarbonyl in studies conducted by Hahn and Charalampopoulos $^{21}$ and Kim et $\mathrm{al}^{22}$. Mn-based additives in diesel fuel have also been shown to have a catalytic effect on the oxidation process which resulted in significant reduction in the PAH emission $^{23}$. A review of metal based additives and their influence on diesel emission can be found here $^{24}$. 
Although fuel additives are a direct source of metal emission found in particulate matter, some of the later studies also emphasized on the identification of trace metals from lubricating oil additives during combustion of fuels and particulate formation ${ }^{25}$. Nickel (Ni) is one of the additives used in lube oil for its tribological properties such as friction reduction and anti-wear behavior ${ }^{17}$. In addition, emission of nickel has also been associated with wear of engine components ${ }^{26}$ such as bearings, valves, gear plating, etc. There are some studies which explore the application of nickel as a catalyst in soot and/or graphene growth from small hydrocarbon precursors. Ozawa et al. ${ }^{27}$ used transition metal complexes including nickelocene during the combustion of benzene and reported higher fullerene yield in soot when additives were used. Prikhod'ko et al. ${ }^{28}$ used catalytic substrates with copper and nickel in the flame zone of premixed propane-oxygen and found nickel substrates to be more efficient for graphene growth. By controlling the angle of orientation amorphous soot was also observed on the nickel substrate. Synthesis of carbon nanotubes from $\underline{\text { small hydrocarbon precursors using chemical vapor deposition (CVD) also extensively uses nickel }}$ $\underline{\text { nanoparticles as catalysts for nucleation }}{ }^{29}$. The mechanism involves gaseous hydrocarbon precursors being decomposed in the substrate surface and providing $\mathrm{C}$ atoms to form hexagonal rings leading to CNT nucleation and growth. The present study intends to address another unexplored scenario where nickel as a trace element in the flame zone interacts with the PAH precursors during the onset of soot formation. The motivation of the present study stems from the question of how $\mathrm{PAH}$ and $\mathrm{Ni}$ interact during the two pathways of soot formation in the beginning of post-flame zone, namely, physical and chemical nucleation. A better understanding of the role of nickel during the incipient soot formation process would assist in developing suitable tools to address unwanted soot emission. The purpose of the study is accomplished by investigating the growth and nucleation of a variety of PAHs in the presence of a small nickel cluster $\left(\mathrm{Ni}_{13}\right)$ at a 
range of low to high temperatures. To analyze and isolate the role of nickel, we presented a comparison of the results from this study with previous work by Mao et al. $\frac{3027}{4}$ on soot formation from homogenous PAHs. The ReaxFF reactive force field method based molecular dynamics (ReaxFF-MD) simulations were employed to describe the dynamic process because of its enhanced accuracy and reasonable computational cost.

\section{Methodology}

\subsection{ReaxFF introduction}

ReaxFF is a bond order ${ }^{31,32}$ dependent empirical force field method that allows modeling of the bond breaking and formation during molecular dynamics simulations. ReaxFF uses the following expression for describing the overall system energy:

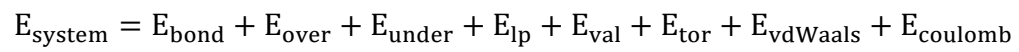

where, the partial energy contributions represent bond, over-coordination penalty, undercoordination stability, lone pair, valence, torsion, non-bonded interactions like van der Waals, and Coulomb energies, respectively.

where, the partial energy contributions represent bond energy ( $E_{\text {bond }}$ ), over-coordination penalty energy $\left(E_{\rho v e r}\right)$, under-coordination stability ( $\left.E_{\text {under }}\right)$, lone pair $\left(E_{d p}\right)$, valence $\left(E_{v a l}\right)$, torsion ( $\left.E_{\text {tor }}\right)$, non-bonded interactions like van der Waals (EvdWaals), and Coulomb (Ecoulomb) energies, respectively.

ReaxFF assumes bond orders are dependent upon bond length and all the connectivitybased interactions i.e. valence and torsion energies are made dependent on bond order. The interatomic distance is the key component in calculating bond orders in ReaxFF. As the distance between molecules change in every iteration, the bond orders are calculated and updated
Field Code Changed

Field Code Changed

Formatted: Subscript

Formatted: Subscript

Formatted: Subscript

Formatted: Subscript

Formatted: Subscript

Formatted: Subscript

Formatted: Subscript

Formatted: Subscript 
accordingly. The effect of bond orders and their energy contributions diminish upon bond breaking. Since ReaxFF is for reactive systems, non-bonded interactions such as van der Waals and Coulomb are usually calculated between each pair of atoms in the system irrespective of their connectivity. However, van der Waals and Coulomb energy expressions use a shielding term to avoid any excessive short distance non-bonded interactions. A connectivity and geometry dependent scheme, Electronegativity Equalization Method $^{3330}$ (EEM) is used for charge calculation. ReaxFF coupled with the molecular dynamics method is much faster than quantumchemical or semi-empirical methods while retaining much of the accuracy of QM methods. Therefore, ReaxFF is particularly suitable for simulations of the complicated reactive events as well as non-reactive ones occurring in large hydrocarbon systems for a long-time scale such as sooting in flame environments.

The ReaxFF Ni/O/C/H description used in this study was developed by incorporating the C description by Srinivasan et al. $\frac{34}{4}$ and the Ni/O description by Zou et al. $\frac{35}{}$ into the Mueller et al. $\mathrm{Ni} / \mathrm{O} / \mathrm{C} / \mathrm{H}$ force field ${ }^{3633}$. The Mueller et al. Ni/C/H/O force field parameters are fitted against the quantum mechanics dataset and have been successfully used to study adsorption and decomposition of hydrocarbons on nickel ${ }^{3734}$. The parameter sets $\mathrm{Ni} / \mathrm{O} / \mathrm{C} / \mathrm{H}$ description can be found in the supporting information.

\subsection{Simulation procedure}

In order to investigate soot nucleation mechanisms from different PAH precursors with wide-ranging molecular weights, we selected six different PAHs that have been identified experimentally in flame temperatures ${ }^{3835}$. The PAHs investigated in this study are naphthalene, anthracene, pyrene, coronene, ovalene and circumcoronene which contain 2-, 3-, 4-, 7-, 10-, and 19- numbered aromatic rings, respectively, with mass ranging between 128 and 666 amu. The
Field Code Changed

Field Code Changed

Field Code Changed

Field Code Changed 
corresponding schematic structures of PAH monomers are displayed in Figure 1. The equilibrium geometry of a $\mathrm{Ni}_{13}$ cluster selected for this study is also shown in Figure 1. The cluster consists of a core nickel atom being surrounded by 12 neighboring Ni atoms. The structure has the shape of

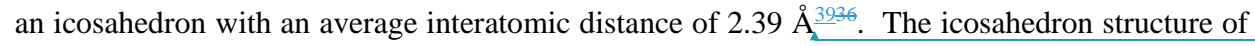
the cluster shows a relatively high binding energy between the $\mathrm{Ni}$ atoms and is more stable than the other members in the small $(\mathrm{N}<30)$ nickel cluster family $\mathrm{An}^{4037}$.

Initially, the structures of each PAH monomer and the nickel cluster are relaxed using the conjugate gradient minimization scheme. After minimization, the nickel cluster is placed at the center of a cubic box of dimension $100 \AA$ x $100 \AA \AA$ x $100 \AA$. Next, 50 duplicates of the minimized PAH monomers are randomly distributed around the nickel cluster. Periodic boundary conditions were employed in all of the three directions. After placement, the whole system is energy minimized and the molecules were brought to a vibrational equilibrium with MD run for $250 \mathrm{ps}$ with a time step of 0.25 fs while the angular and linear momenta are kept to zero. Then, the PAH monomers are given initial velocities generated from the Maxwell-Boltzmann distribution of each temperature, the details of which can be found in a previous study by Mao et al ${ }^{3027}$ Finally, we performed a series of ReaxFF-MD simulations employing the canonical NVT ensemble (constant volume and temperature). To gain a better overview of all the dynamic growth processes during soot nucleation we chose the simulation temperatures as 400, 800, 1200, 1600, 2000 and $2500 \mathrm{~K}$. To control the system temperature, we used the Nose-Hoover thermostat with a damping constant of $100 \mathrm{fs}$. The density of the system is consistent with the homogenous systems used in the previous study and ranges from $0.012 \sim 0.053 \mathrm{~kg} / \mathrm{dm}^{3}$ from the smallest to the largest of the PAHs used in the simulations.

\section{Field Code Changed}

Field Code Changed 


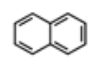

A2

Napthalene

$\left(\mathrm{C}_{10} \mathrm{H}_{8}\right)$

$128 \mathrm{amu}$

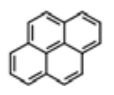

A4

Pyrene $\left(\mathrm{C}_{16} \mathrm{H}_{10}\right)$

$202 \mathrm{amu}$

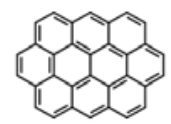

A10

Ovalene $\left(\mathrm{C}_{32} \mathrm{H}_{14}\right)$

$398 \mathrm{amu}$

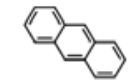

A3

Anthracene $\left(\mathrm{C}_{14} \mathrm{H}_{10}\right)$

$178 \mathrm{amu}$

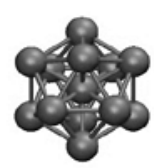

$\mathrm{Ni}_{13}$ Cluster

A7

Coronene $\left(\mathrm{C}_{24} \mathrm{H}_{12}\right)$

$300 \mathrm{amu}$

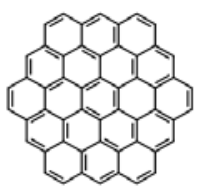

A19

Circumcoronene

$\left(\mathrm{C}_{54} \mathrm{H}_{18}\right)$

$666 \mathrm{amu}$

Figure 1: Structures of the six types of polycyclic aromatic hydrocarbons (PAHs) and the $\mathrm{Ni}_{13}$ cluster studied in ReaxFF MD simulations. The Ni atoms are represented by the grey spheres.

The simulation technique of using high density and high temperature (>1600 K) is

employed to increase the collision frequency of the molecules. The underlying assumption for using high density is that the distribution of collisions between any two molecules in the system is completely random and does not affect subsequent ones. ${ }^{41,42}$ This is a common strategy ${ }_{43,4438,39}^{4 n}$

ReaxFF-MD simulations to accelerate reaction events since the MD time scale ( nanoseconds) is significant order of magnitudes lower than the experimentally observed time-scale of soot formation ( milliseconds $)^{10}$. Furthermore, in order to eliminate statistical noise, we repeated all the case studies for three times with different initial configurations of the molecules in the box. The results are calculated based on the average values obtained from all three simulations. The current MD simulations were performed within the ReaxFF reactive force field program 
implemented in the Amsterdam Density Functional (ADF) software. The trajectory snapshots and movies are generated using Visual Molecular Dynamics (VMD).

\section{Results and Discussion:}

In a homogenous system of PAHs, the transition from gaseous species to solid particles of soot resulted from two distinct pathways $s^{3027}$. At low temperatures, the nonbonded interaction is strong enough for the monomers to form dimers after colliding with each other. The dimers grow in size through further collisions with other monomers/dimers/tetramers and ultimately give rise to condensed soot structures. Another pathway occurs above the decomposition temperature of the PAHs, where chemical reactions involving $\mathrm{PAH}$ fragmentation and reorganization lead to the formation of incipient soot particles. In the following sections 3.1 to 3.3, we investigated to what extent the physical and chemical nucleation of soot particle are affected by the presence of a small nickel cluster. Two PAH monomers are regarded as physically bound dimers when the intermolecular distance is equal to or less than $4 \AA$, the details of which can be found in previous work ${ }^{3027}$. We also discussed the interplay between PAH molecules and the nickel cluster over the temperature range where no significant nucleation occurs. Finally, in section 3.4, we present a comparative analysis of the effect of nickel cluster on the size and carbon-hydrogen ratio of the largest soot particle. We conclude the discussion with a summary of soot nucleation pathways with respect to temperatures and $\mathrm{PAH}$ masses.

\subsection{Physical nucleation}

The mechanism of incipient soot particle formation via physical association of PAHs in the presence of nickel is discussed in this section. Figure 2 shows the key stages of the physical nucleation process for a system containing a $\mathrm{Ni}_{13}$ cluster and 50 ovalene molecules at a temperature of $800 \mathrm{~K}$. Initially, due to random movement of the molecules in the box, the nickel cluster is 
approached by one of the PAH monomers. As the distance between the molecule and the cluster diminishes, the PAH starts to align itself laterally to the surface of the nickel cluster as shown in Figure 2(b). The Ni atoms in the cluster and $\mathrm{C}$ atoms in the PAH molecule bind together and remain closely paired for the rest of the simulation. However, after a while, the pair collides with a second PAH molecule which eventually aligns itself to another face of the $\mathrm{Ni}_{13}$ cluster. Through another collision similar to the ones before, we observe the formation of an incipient soot particle composed of PAH stacks and a single inner layer of three PAH molecules surrounding the $\mathrm{Ni}_{13}$ which acts as the nuclei. The resultant structure is shown in Figure 2(c). This procedure happened within a very short time period of 50 ps. It is noted here that the number of PAHs in the first layer depends on the size of the PAH. Larger PAH molecules leave less area of the $\mathrm{Ni}_{13}$ cluster accessible to other PAH molecules, and thus making it difficult for them to bind to the cluster.

At the same time, random collisions between PAH monomers themselves lead to the formation of several stacked PAH dimers bound by intermolecular interactions. The dimers subsequently collide with other PAH monomers which lead to the formation of trimers or tetramers as observed in the previous study by Mao et al. $\frac{3027}{27}$ As the simulation proceeds, the outer shell of the soot particle comprised of a $\mathrm{Ni}_{13}$ core collides with other PAH stacks (dimers or trimers). With the evolution of time, much larger soot particles having multi-stacked conformations are formed through successful collisions as shown in Figure 2(d) and 2(e). Eventually, we observe two large soot particles, one of them with the $\mathrm{Ni}_{13}$ core, coalesce together to form a final soot particle. During the coalescence, the particle rearranges itself to give rise to a spherical shape while the $\mathrm{Ni}_{13}$ cluster repositions itself to the center. The final soot particle maintains its turbostratic structure ${ }^{40}$ with several locally aligned stacked clusters as observed in the previous study, but with nickel cluster as the core. 


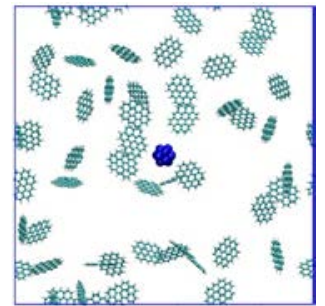

(a) $0.0 \mathrm{ps}$

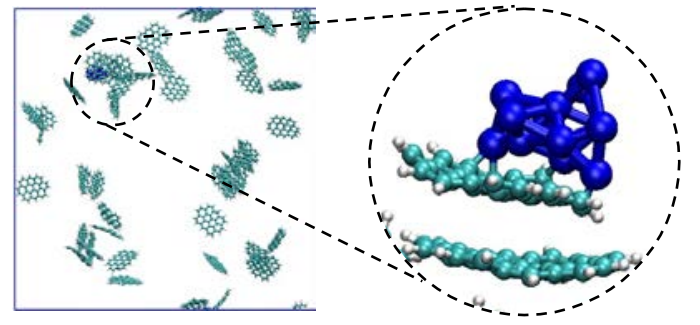

(b) $19.75 \mathrm{ps}$

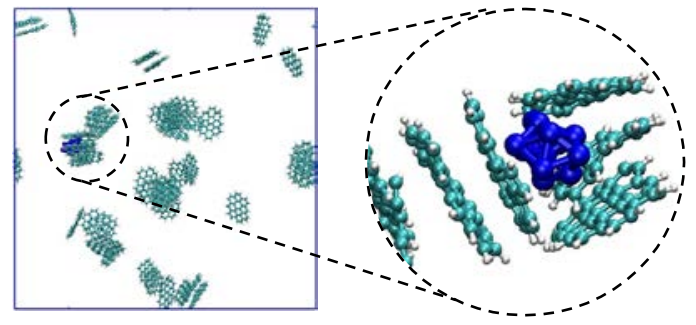

(c) $47.25 \mathrm{ps}$

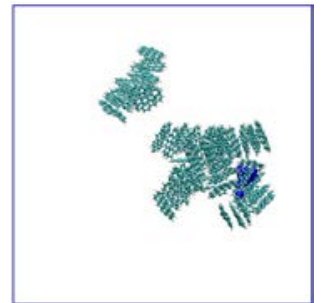

(e) $300.5 \mathrm{ps}$

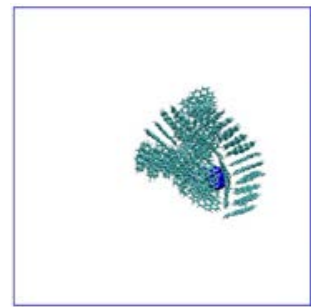

(f) $992.0 \mathrm{ps}$

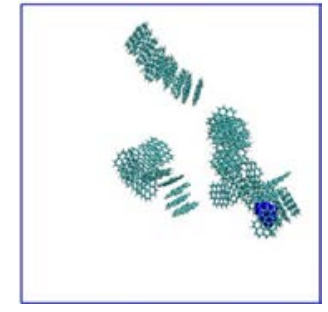

(d) $269.0 \mathrm{ps}$

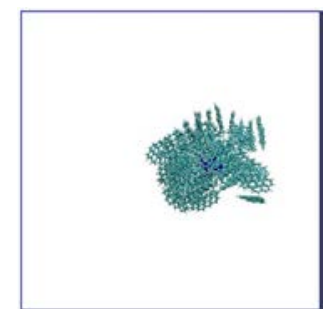

(g) $2000.0 \mathrm{ps}$

Figure 2: A series of snapshots demonstrating physical nucleation of soot particle obtained at different time of the ReaxFF-MD simulations for a system containing 50 ovalene molecules and $\mathrm{Ni}_{13}$ cluster at 800

$\mathrm{K}$. Cyan, white and blue spheres represent carbon, hydrogen and nickel atoms, respectively.

\subsection{No Nucleation}

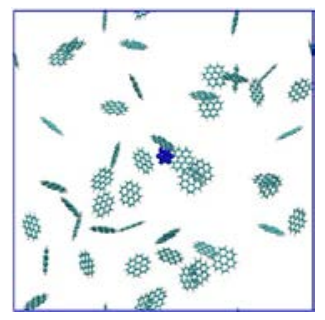

(a) $0.0 \mathrm{ps}$

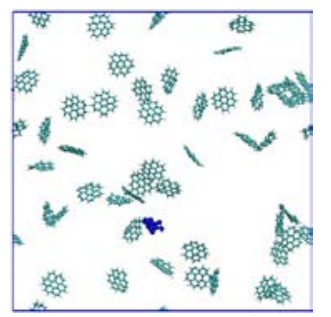

(b) $21.25 \mathrm{ps}$

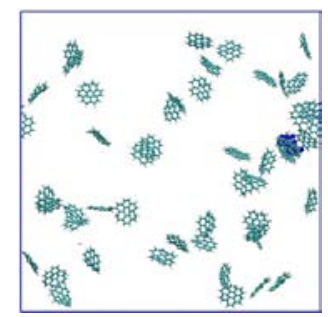

(c) $847.25 \mathrm{ps}$ 


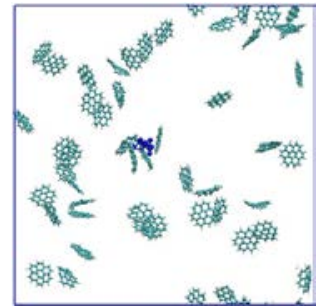

(g) $1185.0 \mathrm{ps}$

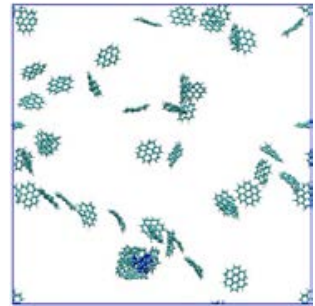

(h) $1662.25 \mathrm{ps}$

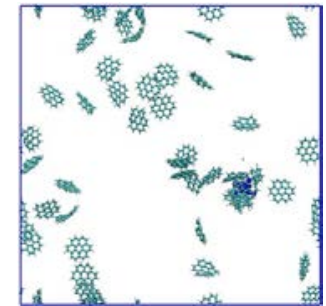

(i) $2000.0 \mathrm{ps}$

Figure 3: A series of snapshots demonstrating no significant nucleation of soot particle obtained at different time of the ReaxFF-MD simulations for a system containing 50 coronene monomers and $\mathrm{Ni}_{13}$ cluster at $1600 \mathrm{~K}$. Cyan, white and blue spheres represent carbon, hydrogen and nickel atoms, respectively.

Absence of physical nucleation or chemical growth in the presence of nickel cluster was observed at moderately high temperatures (1200 1600 K). Figure 3 shows snapshots of the trajectories of 50 coronene molecules at a temperature of $1600 \mathrm{~K}$ obtained through ReaxFF-MD simulations. In a system without nickel clusters we observed the formation of short-lived dimers or trimers $\mathrm{SO27}^{3027}$. At this temperature, the kinetic energy and the number of collisions of the PAHs are higher than those at low temperatures, but the weak binding energy deters the dimers or trimers to grow in size. Hence these bound molecules evaporate back into monomers again before they can collide with other monomers and grow into larger soot particles. However, with the nickel cluster present in the system, we observe some PAHs are bound to the nickel cluster surface with strong $\mathrm{Ni}$ and $\mathrm{C}$ bonding which can survive this temperature. Figures 3(a) to 3(h) show the growth of the small particle as one by one PAH monomers are bound to the nickel surface. Contrary to that at low temperature, growth of the particles is limited to only the first layer outside the $\mathrm{Ni}_{3}$ cluster. The final particle with five PAHs attached to nickel as shown in Figure 3(h) is not sufficiently large compared to typically accepted nascent soot particle size $e^{3027}$ to be considered as condensed soot particle and the region is identified as a no nucleation zone. 


\subsection{Chemical Nucleation}

With the rise of temperature, the weak van der Waals force is not strong enough compared to the high translational kinetic energy of the PAHs to bind them together. In moderately high temperatures, we observed chemisorped PAHs in the nickel cluster. For small PAHs (up to A7) the particle growth was insignificant in size resulting in no nucleation. But at 2000 Kand with extended timespan, we observed PAH chemisorption of nickel which gave rise to stable and condensed particles sufficiently large enough to be considered incipient soot. Figure 4 shows the snapshots of a typical chemisorption process of an ovalene molecule on the nickel cluster. From close observation of Figure 4(a) we found that the PAH molecule initially approaches and binds itself to the distorted icosahedral surface of the nickel cluster laterally. After about 30 ps, the nickel cluster absorbs a hydrogen atom (inside red circle) by breaking a C-H bond as shown in Figure 4(b). The PAH, after losing an $\mathrm{H}$ atom, aligns itself in a perpendicular orientation to the nickel cluster while being surrounded by nickel atoms on both sides. Next, Figure 4(c) shows the cleavage of C-C bond of the partially dehydrogenated PAH molecule by nickel atoms. Similar mechanism is observed when monomers of 2-, 4-, 7- membered aromatic rings are chemisorbed into the nickel cluster leading to the formation of an incipient soot particle. The small size of the cluster barely passes our criterion for a condensed particle to be considered as soot. However, this phenomenon does indicate the possibility to observe nickel-assisted formation of soot at high temperatures without the complete fragmentation of the PAHs. The chemisorption tendency of PAH onto nickel cluster observed in ReaxFF-MD simulations in this work agrees well with previous theoretical and experimental works on chemical deposition on nickel using hydrocarbons $s_{4}^{37,4734,42}$.

Field Code Changed 


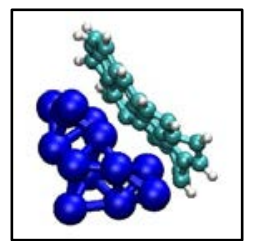

(a) $89.25 \mathrm{ps}$

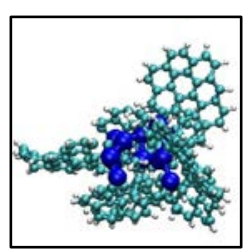

(d) $2000.0 \mathrm{ps}$

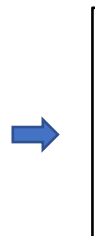

(b)

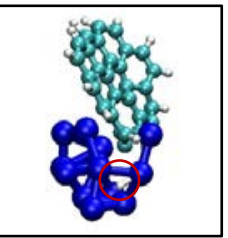

(b) 120.25 ps

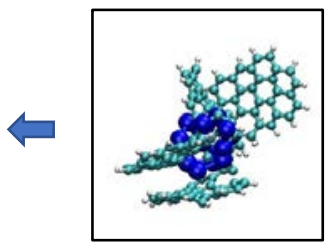

(e) 443.75 ps

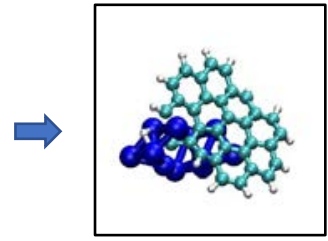

(c) $122.0 \mathrm{ps}$

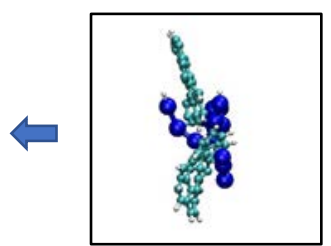

(f) $130.5 \mathrm{ps}$

Figure 4: Snapshots of key stages demonstrating chemisorption of ovalene monomers into a $\mathrm{Ni}_{13}$ cluster at $2000 \mathrm{~K}$ representing incipient soot formation by chemical nucleation obtained from ReaxFF-MD simulations. Cyan, white and blue spheres represent carbon, hydrogen and nickel atoms, respectively.

The purely chemical pathway of nucleation occurs above $2000 \mathrm{~K}$ where the gaseous PAH molecules transform into condensed phase soot particles through the formation of chemical bonds that could survive the high temperature environment. The ReaxFF-MD simulations of homomolecular systems of PAHs indicate two possible routes for growth $\mathrm{h}^{3027}$. The first one is the

\section{Field Code Changed} formation of PAH stacks interconnected by aliphatic chains and the other one is the restructuring of PAH rings to generate fullerene-type carbonaceous soot particles. In our MD simulations, we observed the second route as the dominant pathway for chemical growth of soot at high temperatures. The presence of the nickel cluster is the most likely cause as nickel possesses graphitization properties $\frac{48,4943,44}{4}$. The key stages of the chemical nucleation mechanism for circumcoronene at $2500 \mathrm{~K}$ are shown in Figure 5. In order to have a better understanding of the role of the nickel cluster in facilitating PAH fragmentation and reorganization, we have plotted the time evolution of the number of aromatic rings from three up to eight C-members in Figure 5. 


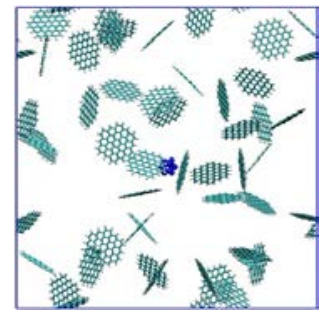

(a) $0.0 \mathrm{ps}$

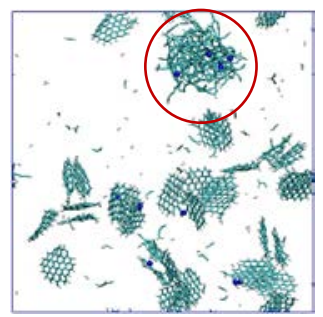

(d) $230.75 \mathrm{ps}$

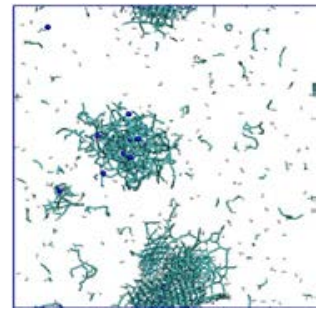

(g) $380.75 \mathrm{ps}$

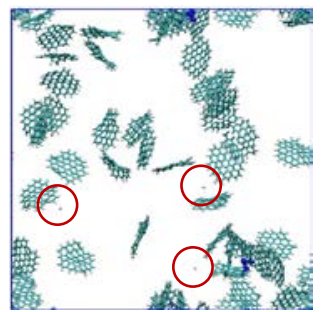

(b) $59.0 \mathrm{ps}$

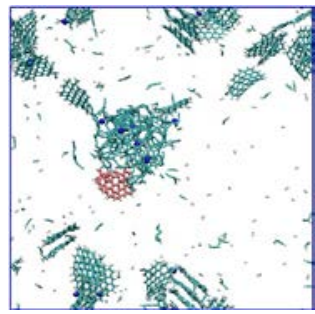

(e) $269.0 \mathrm{ps}$

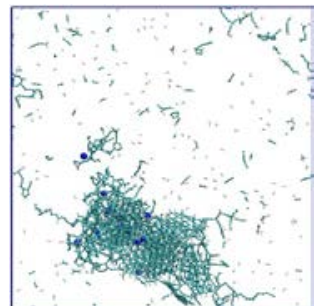

(h) $1407.25 \mathrm{ps}$

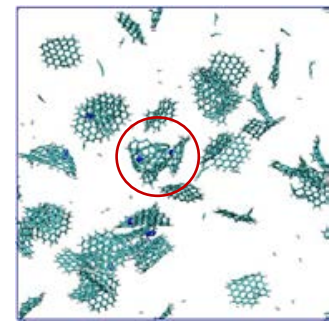

(c) $134.0 \mathrm{ps}$

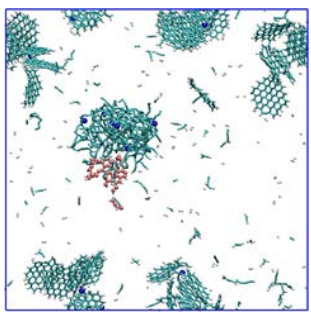

(f) $300.5 \mathrm{ps}$

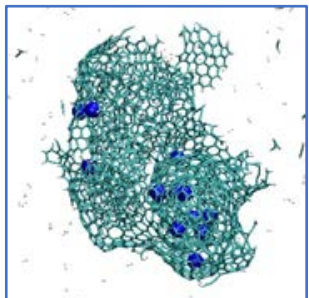

(i) $2000.0 \mathrm{ps}$

Figure 5: Snapshots of key stages of 50 circumcoronene monomers and $\mathrm{Ni}_{13}$ cluster at $2500 \mathrm{~K}$ representing incipient soot formation by chemical nucleation obtained from ReaxFF-MD simulations for. Cyan, white and blue spheres represent carbon, hydrogen and nickel atoms, respectively. The orange color atoms in (e) and (f) show the PAH monomer being chemisorped by the soot cluster.

The overall chemical growth mechanism leading to the formation of polycyclic carbon networks of $\mathrm{PAH}$ monomers in the presence of the nickel cluster remains the same as those observed in a homomolecular system ${ }^{3027}$. However, our MD-simulations indicate that the role of dehydrogenation of PAH monomers as shown in red circles of Figure 5(b). The completely vaporized nickel atoms attach to the carbon atoms of PAH monomers through random collisions. 
Figure 5(c) shows a small soot structure (inside red circle) with bonded nickel atoms which later grows in size by consuming PAH monomers as demonstrated by Figures 5(d)-(f). According to Figure 6, the number of 6 C-member ring starts to decrease at a high rate from approximately 600 to 1400 ps, whereas in the homogenous system this occurs at a later time (1000 1700 ps). The decline indicates the early onset of $\mathrm{C}-\mathrm{C}$ bond splitting and the formation of linear polyyne/polyacetylene chains. At around 1400 ps, we can see from Figures 5 and 6 that the largest cluster is already generated but the number of cyclic rings is at a minimum representing the chaotic and highly disorganized nature of the carbonaceous soot particle.

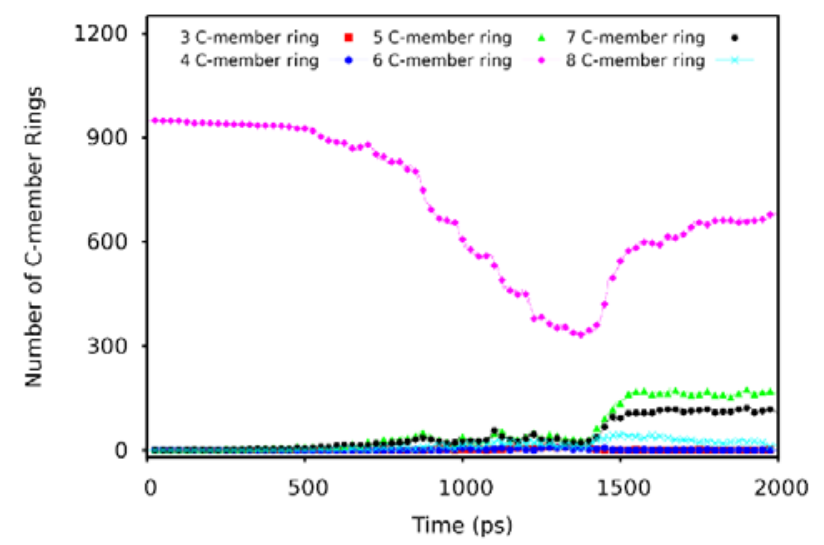

Figure 6: Ring count statistics during the soot nucleation from circumcoronene monomers as a function of simulation time at $2500 \mathrm{~K}$.

After 1400 ps, the numbers of 5,6,7 C-member rings start to increase again due to the internal reorganization of aromatic rings. In a homogenous system of PAHs, restructuring does not occur before 1700 ps. The rearrangement finally leads to a multi-shell fullerenic carbon structure with nickel atoms trapped on the surface of the shells. The final soot particle resembles the structure of endohedral metallofullerenes that has been previously investigated in theoretical and

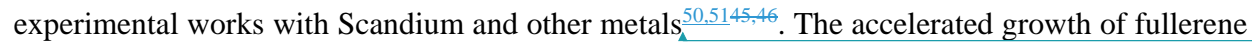


type structure in our MD simulations can be attributed to the presence of nickel which can catalyze graphitization $n^{4742}$ and lower the activation barrier for C-H bond breaking ${ }^{5247}$.

\subsection{Mechanisms of soot nucleation}

The aim of this work is to investigate the incipient soot formation and growth in the context of a small nickel cluster through ReaxFF MD simulations. In this section, we quantitatively discuss the effects of various conditions under which the formation of soot clusters has been studied: PAH molecular weight and system temperature. We compare our ReaxFF-MD results with the previous work by Mao et al. $\underline{\underline{3} 27}$ The clustering data is averaged from three independent simulations with different initial configurations. Figure 7(a) shows the final size of the largest soot particle formed during the ReaxFF-MD simulations of six different PAHs at different temperatures and Figure 7(b) shows the corresponding $\mathrm{C} / \mathrm{H}$ ratios. We have adopted a similar methodology for the quantitative analysis as mentioned in the previous study in order to isolate the effects from $\mathrm{Ni}$ on PAH nucleation. To set up a criterion for soot formation, we considered the threshold monomer number in a nucleated particle as greater than 5 for all the PAHs studied. According to our observations in earlier sections, soot formation through chemical nucleation involves PAH fragmentation, ring-opening and reorganization. Hence to maintain consistency between physical and chemical nucleation pathways we divided the cluster mass by the corresponding monomer mass to convert it into an equivalent number of monomers present in the cluster. In all cases however, the mass of the nickel cluster has not been taken into consideration during the calculation of the size of the largest soot particle.

The most noticeable effect of $\mathrm{Ni}_{13}$ cluster on soot nucleation can be seen at low temperatures when physical nucleation through sticking collisions is the only pathway for the PAH molecules to form large soot particles. At $400 \mathrm{~K}$, all of the homomolecular systems contained
Field Code Changed

Field Code Changed

Field Code Changed 
several distinct soot particles and the largest cluster formed in those systems comprised of 39-64\% of all the PAH molecules present in the system. But in the presence of nickel cluster, the results demonstrate a stark increase in the size of the largest cluster. The final soot particle after the simulations contained about $82-100 \%$ of all the PAH molecules present in the system. The cause for the overall increase in the size of the cluster is the site provided by the nickel cluster during the simulations. Initially the PAHs bind around the surface of the nickel cluster, thus providing threedimensional surface area for further collision. This early onset of nucleation paves the way for the PAHs to bind and form larger clusters within the simulation lifetime.

When the temperature is raised to $800 \mathrm{~K}$, the nickel cluster presence shows very limited effect on cluster sizes. For smaller PAHs like naphthalene, anthracene and pyrene with weak intermolecular interactions, we see a slight increase of about 1 3 molecules in the cluster. This is owing to the strong bonding between the PAHs and the nickel cluster that could survive this temperature. Larger molecules like coronene, ovalene and circumcoronene, have stronger intermolecular interactions and more successful collisions at this temperature ${ }_{4148}^{4}$. Thus, in these

Field Code Changed cases the elevated temperature and increased number of sticking collisions result in the formation of large soot particles without the help of the nickel cluster. Between $1200 \mathrm{~K}$ and $1600 \mathrm{~K}$, there is only slight difference in size between the two systems for PAH molecules up to coronene. However, for some cases, i.e. coronene at $1200 \mathrm{~K}$, the addition of nickel resulted in a cluster large enough (containing greater than 5 monomers) to be considered incipient soot particle. All of the PAH molecules manifest a similar trend at $2000 \mathrm{~K}$, where the dehydrogenation and chemisorption of PAH monomers by nickel gives rise to nascent soot, resulting in lowering the chemical nucleation region from $2500 \mathrm{~K}^{3027}$ to $2000 \mathrm{~K}$.

Field Code Changed 


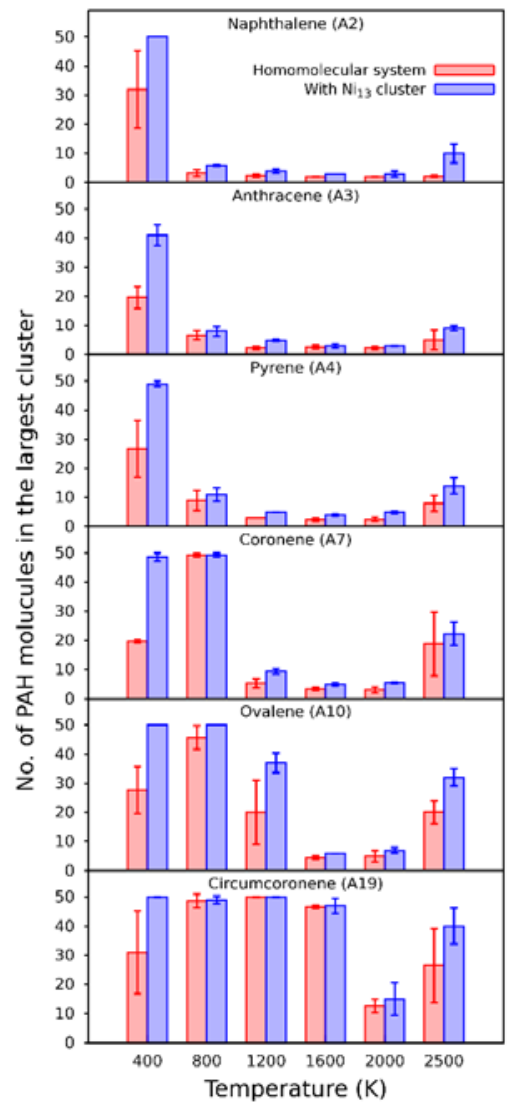

(a)

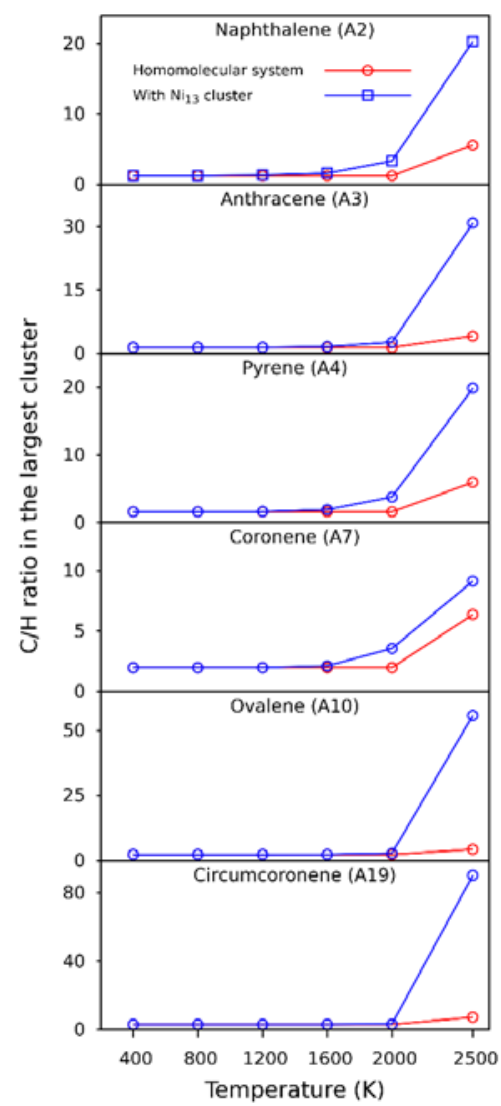

(b)

Figure 7: ReaxFF-MD results of (a) equivalent PAH monomer numbers and (b) $\mathrm{C} / \mathrm{H}$ ratio in the largest soot particle formed within systems of six different PAHs, namely naphthalene (A2), anthracene (A3), pyrene (A4), coronene (A7), ovalene (A10) and circumcoronene (A19) and $\mathrm{Ni}_{13}$ cluster as a function of temperature ranging from 400 to $2500 \mathrm{~K}$.

At $2500 \mathrm{~K}$, all the PAHs become chemically active and we again observe the formation and growth of incipient soot particle. Our MD results indicated longer polycyclic or chain structures for naphthalene, anthracene and pyrene systems compared to homomolecular systems. However, the graphitization property of nickel is most prominently observed in large PAH systems. The presence of nickel brought about the early initiation of ring-opening, formation of 
large carbon networks and internal redistribution of 5-, 6- and 7-membered rings to form bigger three-dimensional caged structures than those observed in homogenous systems of PAHs. The extent of nickel assisted C-H bond breaking can also be observed in Figure 7(b). At temperatures below $2000 \mathrm{~K}$, the $\mathrm{C} / \mathrm{H}$ ratios of the largest soot particles in homogenous systems are the same as the corresponding PAH monomers. However, with nickel, the dehydrogenation begins at temperatures as early as $1200 \mathrm{~K}$ for smaller PAHs. For larger molecules, the $\mathrm{C} / \mathrm{H}$ ratio increases after $1600 \mathrm{~K}$. At $2500 \mathrm{~K}$, we observe a significant rise in the $\mathrm{C} / \mathrm{H}$ ratio of all the PAHs in the nickel cluster system. The results confirm the catalytic role of nickel in dehydrogenation and facilitating the process of fullerene type soot formation.

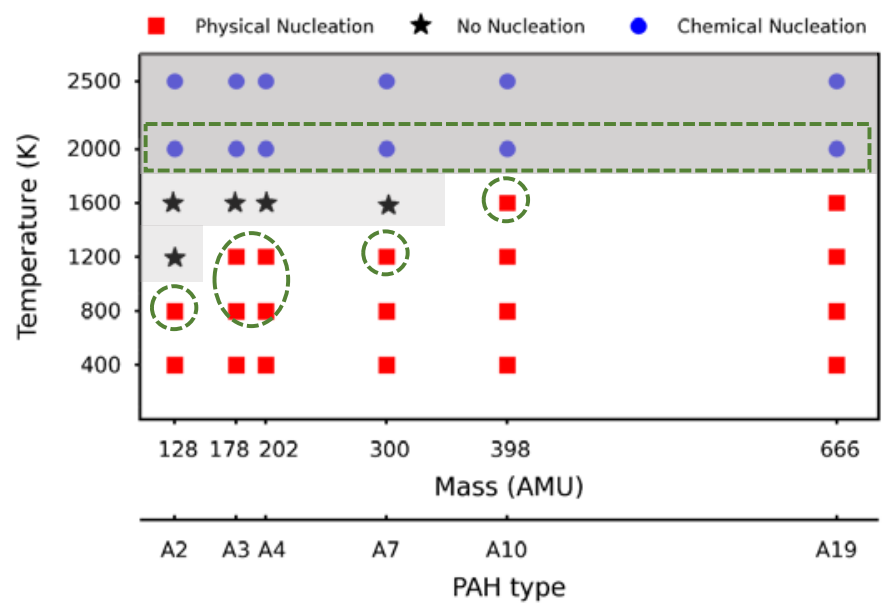

Figure 8: Overall representation of three different nucleation pathways of soot formation from PAH precursors in the presence of a $\mathrm{Ni}_{13}$ cluster at a range of temperatures from $400 \mathrm{~K}$ to $2500 \mathrm{~K}$. The physical nucleation, no nucleation and chemical nucleation pathways are denoted by closed red squares, black diamonds and blue circles, respectively. Depending upon the PAH type and mass and temperature, the three regimes of soot nucleation are shown as: (i) physical nucleation (white), (ii) no nucleation (light grey) and (iii) chemical nucleation (dark grey).

A summary of PAH soot formation behavior is presented in Figure 8, where three distinct regions of sooting mechanism has been identified with white (physical nucleation), light grey (no 
nucleation) and dark grey (chemical nucleation). The green dashed zones indicate the temperatures at which we observed deviation from previously studied homogenous soot nucleation behaviors. The overall effect of the nickel cluster is twofold: first, to raise the physical nucleation region for naphthalene, anthracene, pyrene, coronene and ovalene and second, to lower the chemical nucleation region for all the PAHs thus shrinking the no nucleation area, with respect to temperature.

\section{Conclusion}

In this paper, we have investigated the effect of a small nickel cluster $\left(\mathrm{Ni}_{13}\right)$ on the dynamic processes of soot formation and growth using ReaxFF-MD simulations. We scrutinized the nucleation process of incipient soot particles from six different PAH monomers, namely, naphthalene $\left(\mathrm{C}_{10} \mathrm{H}_{8}\right)$, anthracene $\left(\mathrm{C}_{14} \mathrm{H}_{10}\right)$, pyrene $\left(\mathrm{C}_{16} \mathrm{H}_{10}\right)$, coronene $\left(\mathrm{C}_{24} \mathrm{H}_{12}\right)$, ovalene $\left(\mathrm{C}_{32} \mathrm{H}_{14}\right)$ and circumcoronene $\left(\mathrm{C}_{54} \mathrm{H}_{18}\right)$, constituted of 2-, 3-, 4-, 7-, 10-, and 19-numbered aromatic rings, respectively. To determine the role of the nickel cluster, we have compared the results of this study

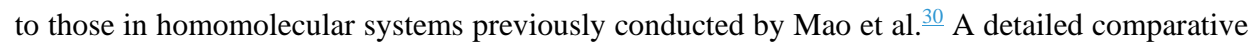
description of the mechanisms of soot formation in those two systems through physical and chemical nucleation as well as a quantitative analysis of the incipient soot particles is presented in the study. The temperature effects on the nucleation pathways and the final soot size have been obtained by performing a series of ReaxFF-MD simulations for a range of temperatures from 400 $\mathrm{K}$ to $2500 \mathrm{~K}$.

At low temperatures such as $400 \mathrm{~K}$ and $800 \mathrm{~K}$ soot formation is through physical nucleation only. Aside from colliding and forming dimers and trimers, the PAHs attach themselves to the nickel cluster surface which acts as a nucleus and collide with other monomers and form incipient particles comprising stacked clusters around nickel. The turbostratic cluster thus formed comprises 
of PAH molecules bound by van der Waals forces with the nickel cluster claiming the center. Since the PAHs attached to the nickel cluster provides more surface area for soot growth than dimers or trimers, we observe the formation of larger soot particles during the same time span as compared to the homomolecular system. At an elevated temperature such as $800 \mathrm{~K}$, the effect of the nickel cluster is not very significant for larger PAHs. Only in the systems of smaller PAHs we observe an overall addition of 1 3 monomers to the largest cluster leading to soot particle formation. Even at high temperatures with increased collision frequency, binding energies between larger PAH molecules are strong enough to produce soot particles without any help of a nickel cluster. Within the temperature range of $1200 \mathrm{~K}$ to $2000 \mathrm{~K}$, we observe chemisorption of PAH molecules onto the nickel cluster. The dehydrogenated and chemisorped monomers cause soot formation via the chemical route at an early temperature of $2000 \mathrm{~K}$.

At a high temperature such as $2500 \mathrm{~K}$, the dominant chemical pathway is the generation of the fullerene type soot particle. This is because of the catalytic role of the nickel cluster in hydrogen removal and $\mathrm{C}-\mathrm{C}$ bond fission of the PAHs while forming large carbon networks. The simulations conducted in this study suggest the presence of nickel clusters cause early dehydrogenation and C$\mathrm{C}$ bond breaking and thus accelerates the ring opening, fragmentation, and later internal rearrangement to form the fullerenic structure. The comparison of time evolution of C-member ring numbers in the homomolecular system and a system with nickel cluster confirms that the reorganization and condensation occur earlier in the latter case. The final soot particle is an endohedral type multi-shell fullerene structure with nickel atoms at the surface of the cage. The atomic level understanding details obtained from ReaxFF-MD results from of this study paves the way towards gaining more insight into-facilitate the study on the complex mechanisms of soot formation and growth in the presence of metal clusters-which can have further practical 
implications. The approach presented here provides valuable insights into the prediction of sooting tendency with regard to trace metal elements, which are ubiquitous in the combustion chamber and as such are of major importance. Reduction of particulate matter emissions is a significant factor to consider during the design of diesel engines. Additional insights into the design and how the trace metals affect the growth of soot can have potential to control this type of pollutant.

\section{Conflicts of Interest}

The authors declare no conflicts of interest.

\section{Acknowledgement}

SS and ACTvD acknowledge funding by AFOSR grant FA9550-14-1-0355 and from NSF grant\#1462980. QM acknowledges funding from the Major Programmes of the National Science

Foundation of China (Grant No. 51390493 and Grant No. 91441120) and the China Scholarship

Council. KHL acknowledges funding from the "UK Consortium on Mesoscale Engineering

Sciences (UKCOMES)” (EPSRC Grant Nos. EP/L00030X/1 and EP/R029598/1).

\section{References:}

(1) Jiménez-Espadafor F. J.; Torres M.; Velez J. A.; Carvajal E.; Becerra J. A. Experimental Analysis Of Low Temperature Combustion Mode With Diesel And Biodiesel Fuels: A Method For Reducing NOx And Soot Emissions. Fuel Process. Technol. 2012, 103, pp. 57-63.

(2) Nowack B.; Bucheli T. D. Occurrence, Behavior And Effects Of Nanoparticles In The Environment. Environ. Pollut. 2007, 150(1), pp. 5-22.

(3) Dockery D. W. Epidemiologic Evidence Of Cardiovascular Effects Of Particulate Air Pollution. Environ. Health Perspect. 2001, 109 Suppl 4, pp. 483-6.

(4) Pope C. A.; Burnett R. T.; Thun M. J.; Calle E. E.; Krewski D.; Ito K.; et al. Lung Cancer, Cardiopulmonary Mortality, And Long-Term Exposure To Fine Particulate Air Pollution. JAMA 2002, 287(9), pp. 1132-41.

(5) Kelly F. J.; Fussell J. C. Air Pollution And Public Health: Emerging Hazards And Improved Understanding Of Risk. Environ. Geochem. Health 2015, 37(4), pp. 631-49. 
(6) Nadimpalli N. K. V.; Buddhiraju V. S.; Runkana V. Modeling And Simulation Of Carbon Black Synthesis In An Aerosol Flame Reactor. Adv. Powder Technol. 2011, 22(1), pp. 141$\underline{9 .}$

(7) Lau X. C.; Wang Z.; Mitra S. Effect Of Low Concentrations Of Carbon Black In Organic Solar Cells. Sol. Energy Mater. Sol. Cells 2014, 128, pp. 69-76.

(8) Richter H.; Howard J. B. Formation Of Polycyclic Aromatic Hydrocarbons And Their Growth To Soot-A Review Of Chemical Reaction Pathways. Prog. Energy Combust. Sci. 2000, 26(4), pp. 565-608.

(9) Frenklach M. Reaction Mechanism Of Soot Formation In Flames. Phys. Chem. Chem. Phys. 2002, 4(11), pp. 2028-37.

(10) Wang H. Formation Of Nascent Soot And Other Condensed-Phase Materials In Flames. Proc. Combust. Inst. 2011, 33(1), pp. 41-67.

(11) Herdman J. D.; Miller J. H. Intermolecular Potential Calculations For Polynuclear Aromatic Hydrocarbon Clusters. J. Phys. Chem. A 2008, 112(28), pp. 6249-56.

(12) Frenklach M.; Wang H. Detailed Mechanism and Modeling of Soot Particle Formation. In: Bockhorn PDH, editor. Soot Formation in Combustion. Springer Berlin Heidelberg; 1994. p. 165-92.

(13) Lough G. C.; Schauer J. J.; Park J.-S.; Shafer M. M.; DeMinter J. T.; Weinstein J. P. Emissions Of Metals Associated With Motor Vehicle Roadways. Environ. Sci. Technol. 2005, 39(3), pp. 826-36.

(14) Lombaert K.; Morel S.; Le Moyne L.; Adam P.; Tardieu de Maleissye J.; Amouroux J. Nondestructive Analysis Of Metallic Elements In Diesel Soot Collected On Filter: Benefits Of Laser Induced Breakdown Spectroscopy. Plasma Chem. Plasma Process. 2004, 24(1), pp. 41-56.

(15) Corbin J. C.; Mensah A. A.; Pieber S. M.; Orasche J.; Michalke B.; Zanatta M.; et al. Trace Metals In Soot And PM2.5 From Heavy-Fuel-Oil Combustion In A Marine Engine. Environ. Sci. Technol. 2018, 52(11), pp. 6714-22.

(16) Kannan G. R.; Karvembu R.; Anand R. Effect Of Metal Based Additive On Performance Emission And Combustion Characteristics Of Diesel Engine Fuelled With Biodiesel. Appl. Energy 2011, 88(11), pp. 3694-703.

(17) Singh S. K.; Agarwal A. K.; Sharma M. Experimental Investigations Of Heavy Metal Addition In Lubricating Oil And Soot Deposition In An EGR Operated Engine. Appl. Therm. Eng. 2006, 26(2), pp. 259-66.

(18) Wang Y.-F.; Huang K.-L.; Li C.-T.; Mi H.-H.; Luo J.-H.; Tsai P.-J. Emissions Of Fuel Metals Content From A Diesel Vehicle Engine. Atmos. Environ. 2003, 37(33), pp. 4637 $\underline{43 .}$

(19) Hirasawa T.; Sung C.-J.; Yang Z.; Joshi A.; Wang H. Effect Of Ferrocene Addition On Sooting Limits In Laminar Premixed Ethylene-Oxygen-Argon Flames. Combust. Flame 2004, 139(4), pp. 288-99.

(20) Ritrievi K. E.; Longwell J. P.; Sarofim A. F. The Effects Of Ferrocene Addition On Soot Particle Inception And Growth In Premixed Ethylene Flames. Combust. Flame 1987, 70(1), pp. 17-31.

(21) Hahn DW, Charalampopoulos TT. The role of iron additives in sooting premixed flames. Proc Combust Inst 1992; 24: 1007-1014.

(22) Kim K. B.; Masiello K. A.; Hahn D. W. Reduction Of Soot Emissions By Iron Pentacarbonyl In Isooctane Diffusion Flames. Combust. Flame 2008, 154(1), pp. 164-80. 
(23) Yang H.-H.; Lee W.-J.; Mi H.-H.; Wong C.-H.; Chen C.-B. PAH Emissions Influenced By Mn-Based Additive And Turbocharging From A Heavy-Duty Diesel Engine. Environ. Int. 1998, 24(4), pp. 389-403.

(24) Ribeiro N. M.; Pinto A. C.; Quintella C. M.; da Rocha G. O.; Teixeira L. S. G.; Guarieiro L. L. N.; et al. The Role Of Additives For Diesel And Diesel Blended (Ethanol Or Biodiesel) Fuels: A Review. Energy Fuels 2007, 21(4), pp. 2433-45.

(25) Miller A. L.; Stipe C. B.; Habjan M. C.; Ahlstrand G. G. Role Of Lubrication Oil In Particulate Emissions From A Hydrogen-Powered Internal Combustion Engine. Environ. Sci. Technol. 2007, 41(19), pp. 6828-35.

(26) Dwivedi D.; Agarwal A. K.; Sharma M. Particulate Emission Characterization Of A Biodiesel Vs Diesel-Fuelled Compression Ignition Transport Engine: A Comparative Study. Atmos. Environ. 2006, 40(29), pp. 5586-95.

(27) Ozawa M.; Osawa E. Additives To Improve The Yield Of Fullerenes From The Combustion Of Benzene. Carbon 1999, 37(4), pp. 707-9.

(28) Prikhod'ko N. G.; Lesbaev B. T.; Auelkhankyzy M.; Mansurov Z. A. Synthesis Of Graphene Films In A Flame. Russ. J. Phys. Chem. B 2014, 8(1), pp. 61-4.

(29) Moors M.; Amara H.; Visart de Bocarmé T.; Bichara C.; Ducastelle F.; Kruse N.; et al. Early Stages In The Nucleation Process Of Carbon Nanotubes. ACS Nano 2009, 3(3), pp. $\underline{511-6 .}$

(30) Mao Q.; van Duin A. C. T.; Luo K. H. Formation Of Incipient Soot Particles From Polycyclic Aromatic Hydrocarbons: A ReaxFF Molecular Dynamics Study. Carbon 2017, 121, pp. 380-8.

(31) Brenner D. W. Empirical Potential For Hydrocarbons For Use In Simulating The Chemical Vapor Deposition Of Diamond Films. Phys. Rev. B 1990, 42(15), pp. 9458-71.

(32) Tersoff J. Empirical Interatomic Potential For Carbon, With Applications To Amorphous Carbon. Phys. Rev. Lett. 1988, 61(25), pp. 2879-82.

(33) Mortier W. J.; Ghosh S. K.; Shankar S. Electronegativity-Equalization Method For The Calculation Of Atomic Charges In Molecules. J. Am. Chem. Soc. 1986, 108(15), pp. 431520.

(34) Srinivasan S. G.; van Duin A. C. T.; Ganesh P. Development Of A ReaxFF Potential For Carbon Condensed Phases And Its Application To The Thermal Fragmentation Of A Large Fullerene. J. Phys. Chem. A 2015, 119(4), pp. 571-80.

(35) Zou C.; Shin Y. K.; van Duin A. C. T.; Fang H.; Liu Z.-K. Molecular Dynamics Simulations Of The Effects Of Vacancies On Nickel Self-Diffusion, Oxygen Diffusion And Oxidation Initiation In Nickel, Using The ReaxFF Reactive Force Field. Acta Mater. 2015, 83, pp. 102-12.

(36) Mueller J. E.; van Duin A. C. T.; Goddard W. A. Development And Validation Of ReaxFF Reactive Force Field For Hydrocarbon Chemistry Catalyzed By Nickel. J. Phys. Chem. C 2010, 114(11), pp. 4939-49.

(37) Mueller J. E.; van Duin A. C. T.; Goddard W. A. Application Of The ReaxFF Reactive Force Field To Reactive Dynamics Of Hydrocarbon Chemisorption And Decomposition. $J$. Phys. Chem. C 2010, 114(12), pp. 5675-85.

(38) Happold J.; Grotheer H.-H.; Aigner M. Distinction Of Gaseous Soot Precursor Molecules And Soot Precursor Particles Through Photoionization Mass Spectrometry. Rapid Commun. Mass Spectrom. RCM 2007, 21(7), pp. 1247-54. 
(39) Nayak S. K.; Khanna S. N.; Rao B. K.; Jena P. Physics Of Nickel Clusters: Energetics And Equilibrium Geometries. J. Phys. Chem. A 1997, 101(6), pp. 1072-80.

(40) Stave M. S.; DePristo A. E. The Structure Of NiN And PdN Clusters: $4 \leq \mathrm{N} \leq 23$. J. Chem. Phys. 1992, 97(5), pp. 3386-98.

(41) Totton T. S.; Misquitta A. J.; Kraft M. A Quantitative Study Of The Clustering Of Polycyclic Aromatic Hydrocarbons At High Temperatures. Phys. Chem. Chem. Phys. 2012, 14(12), pp. 4081-94.

(42) Chung S.-H.; Violi A. Peri-Condensed Aromatics With Aliphatic Chains As Key Intermediates For The Nucleation Of Aromatic Hydrocarbons. Proc. Combust. Inst. 2011, 33(1), pp. 693-700.

(43) Chenoweth K.; van Duin A. C. T.; Goddard W. A. ReaxFF Reactive Force Field For Molecular Dynamics Simulations Of Hydrocarbon Oxidation. J. Phys. Chem. A 2008, 112(5), pp. 1040-53.

(44) Castro-Marcano F.; Kamat A. M.; Russo M. F.; van Duin A. C. T.; Mathews J. P. Combustion Of An Illinois No. 6 Coal Char Simulated Using An Atomistic Char Representation And The ReaxFF Reactive Force Field. Combust. Flame 2012, 159(3), pp. 1272-85.

(45) Yan S.; Jiang Y.-J.; Marsh N. D.; Eddings E. G.; Sarofim A. F.; Pugmire R. J. Study Of The Evolution Of Soot From Various Fuels. Energy Fuels 2005, 19(5), pp. 1804-11.

(46) Clague A. D. H.; Donnet J. B.; Wang T. K.; Peng J. C. M. A Comparison Of Diesel Engine Soot With Carbon Black11Presented At The 1997 UK Carbon Group Meeting "Current UK Research In Carbon Science Technology” (M. Turpin And J. Patrick, Organisers). Carbon 1999, 37(10), pp. 1553-65.

(47) Lu Y.; Yang X. Molecular Simulation Of Graphene Growth By Chemical Deposition On Nickel Using Polycyclic Aromatic Hydrocarbons. Carbon 2015, 81, pp. 564-73.

(48) Ōya A.; Ōtani S. Catalytic Graphitization Of Carbons By Various Metals. Carbon 1979, 17(2), pp. 131-7.

(49) Ōya A.; Mochizuki M.; Ōtani S.; Tomizuka I. An Electron Microscopic Study On The Turbostratic Carbon Formed In Phenolic Resin Carbon By Catalytic Action Of Finely Dispersed Nickel. Carbon 1979, 17(1), pp. 71-6.

(50) Chen N.; Chaur M. N.; Moore C.; Pinzón J. R.; Valencia R.; Rodríguez-Fortea A.; et al. Synthesis Of A New Endohedral Fullerene Family, Sc2S@C2n (N = 40-50) By The Introduction Of SO2. Chem. Commun. 2010, 46(26), pp. 4818-20.

(51) Deng Q.; Heine T.; Irle S.; A. Popov A. Self-Assembly Of Endohedral Metallofullerenes: A Decisive Role Of Cooling Gas And Metal-Carbon Bonding. Nanoscale 2016, 8(6), pp. 3796-808.

(52) Liu B.; Lusk M. T.; Ely J. F. Influence Of Nickel Catalyst Geometry On The Dissociation Barriers Of H2 And CH4: Ni13 Versus Ni(111). J. Phys. Chem. C 2009, 113(31), pp. $\underline{13715-22 .}$ 